\title{
Association Between Coronary Artery Ostial Diameter Ratio and Stenotic Coronary Artery Disease in Patients Undergoing Elective Coronary Angiography
}

\section{Mehmet Eyüboğlu1, ๑ Canan Eyüboğlu2}

${ }^{1}$ Gaziosmanpaşa University Faculty of Medicine, Department of Cardiology, Tokat, Turkey

${ }^{2}$ Gaziosmanpaşa University Faculty of Medicine, Department of Anatomy, Tokat, Turkey

\begin{abstract}
Objectives: Besides the traditional cardiovascular risk factors, there are numerous anatomical and functional parameters that affect the coronary atherosclerosis and subsequent stenotic coronary artery disease (CAD). Previous studies reported a significant association between coronary artery diameter and stenotic CAD. However, no study has investigated the association between the ratio of ostial diameter of the main coronary arteries and stenotic CAD. We aimed to investigate whether coronary artery ostial diameter ratio (CAOD-R), which is calculated as dividing the left main coronary artery (LMCA) ostial diameter by the right coronary artery (RCA) ostial diameter, was associated with the presence of stenotic $\mathrm{CAD}$ in patients undergoing elective coronary angiography.

Materials and Methods: Four hundred and thirty-six patients who underwent elective coronary angiography were included in the study. CAOD-R was calculated from digital angiograms for all patients, and patients were divided into two groups as CAOD-R $\leq 1$ or $>1$. The groups were compared regarding the frequency of patients with stenotic CAD, and the association of CAOD-R with the presence of stenotic CAD was investigated.
\end{abstract}

Results: According to calculated CAOD-R, 142 (32.6\%) patients were in the CAOD-R $\leq 1$ group and 294 (67.4\%) patients were in the CAOD-R $>1$ group. Patients with CAOD-R $\leq 1$ had significantly higher frequency of stenotic CAD

Address for Correspondence: Mehmet Eyüboğlu, Gaziosmanpaşa University Faculty of Medicine, Department of Cardiology, Tokat, Turkey e-mail: mhmtybgl@gmail.com ORCID: orcid.org/0000-0002-5754-9382

Received: 07.09.2020 Accepted: 13.11.2020

Cite this article as: Eyüboğlu M, Eyüboğlu C. Association Between Coronary Artery Ostial Diameter Ratio and Stenotic Coronary Artery Disease in Patients Undergoing Elective Coronary Angiography. EJCM 2020;8(4):193-198.

DOI: 10.32596/ejcm.galenos.2020.09.046 


\section{Abstract}

compared to patients with CAOD-R $>1(61.9 \%$ vs $41.1 \%, \mathrm{p}<0.001)$. In the subgroup analysis of patients with stenotic $\mathrm{CAD}$, while the frequency of left anterior descending (LAD) artery stenosis was significantly higher in the CAOD-R $\leq 1$ patient group ( $46.6 \%$ vs $38.8 \%, p=0.006$ ), the frequency of RCA stenosis was significantly higher in the CAOD-R $>1$ patient group $(28.9 \%$ vs $18.2 \%, p=0.002)$. There were no differences between the groups regarding the frequency of stenotic lesions in LMCA and left circumflex artery. Furthermore, multivariate analysis demonstrated that CAOD-R was an independent predictor of stenotic CAD (Odds ratio: 0.824, 95\% confidence interval: 0.721-0.947, $\mathrm{p}<0.001$ ).

Conclusion: The present study demonstrated that CAOD-R was an independent predictor of stenotic CAD in patients undergoing elective coronary angiography. As an easily calculated parameter from angiographic images, CAOD-R may be useful in the further risk assessment of CAD patients undergoing elective coronary angiography.

Keywords: Coronary artery disease, coronary artery diameter, coronary angiography, coronary artery ostial diameter ratio

\section{Introduction}

Atherosclerosis is a progressive disease that has a complex pathophysiology and generally manifests as narrowing of the arteries ${ }^{(1)}$. Coronary artery disease (CAD) is the life-threatening consequence of the atherosclerosis of the coronary vasculature, and despite the considerable improvements in the both diagnostic and therapeutic methods, it remains as a major reason for morbidity and mortality worldwide ${ }^{(2)}$. Chronic coronary syndrome (CCS), previously named as stable CAD, is the main manifestation of the coronary atherosclerosis and includes the majority of patients with $\mathrm{CAD}^{(3)}$. From inflammation to endothelial dysfunction, numerous factors play roles in the occurrence of $\mathrm{CAD}$; however, traditional major cardiovascular risk factors such as age, male gender, smoking, hypercholesterolemia, hypertension and diabetes mellitus (DM) are considered as main triggers of both beginning and progression of the coronary atherosclerosis that leads $\mathrm{CCS}^{(3,4)}$. Nevertheless, CAD has a complex pathophysiology and mechanism, and interaction between clinical and anatomical parameters may take a role in the emergence or acceleration of coronary atherosclerosis, regardless of traditional cardiovascular risk factors ${ }^{(5)}$.

Variations in the anatomy of the coronary vasculature, in particular coronary artery size may be associated with the occurrence of stenotic $\mathrm{CAD}$, and previous reports indicated a significant inverse association between coronary artery diameter and CAD severity ${ }^{(6,7)}$. However, no study has investigated the relationship between ostial diameter ratio of the main coronary arteries and stenotic CAD. Therefore, in the present study, we aimed to investigate whether angiographically detected coronary artery ostial diameter ratio (CAOD-R), which is defined as dividing the ostial diameter of left main coronary artery (LMCA) to ostial diameter of right coronary artery (RCA), predicted stenotic $\mathrm{CAD}$ in the epicardial coronary arteries.

\section{Materials and Methods}

\section{Study Population}

The medical records of 451 consecutive CCS patients who underwent elective coronary angiography between January 2018 and June 2020 were retrospectively screened for the study. Among patients screened, 15 patients were excluded (11 patients for insufficient visualization of coronary artery ostium due to difficulties in the cannulation of coronary ostium and non-selective injection of the contrast media, three patients for ostial right coronary artery (RCA) lesion and one patient for ostial LMCA lesion) from the study. All in all, the remaining 436 patients were included in the study. As a part of routine 
examination, all patients underwent a cardiac and systemic physical examination. A detailed echocardiography was performed to all study participants and a surface 12lead electrocardiography was obtained from patients. Laboratory analyses were made from venous blood samples after an overnight fasting. Hypertension was defined as systolic blood pressure levels of $\geq 140 \mathrm{mmHg}$ and/or diastolic blood pressure levels of $\geq 90 \mathrm{mmHg}$ and/ or known treatment with antihypertensive medications. DM was defined as $\geq 2$ fasting plasma glucose levels of $\geq 126 \mathrm{mg} / \mathrm{dL}$, or 2-hour plasma glucose levels of $\geq 200 \mathrm{mg} /$ $\mathrm{dL}$, or glycated hemoglobin (HbA1c) levels of $\geq 6.5 \%$, or documented treatment with antidiabetic drugs. Previous CAD was defined based on prior angiographic reports and/or documentation of myocardial ischemia on noninvasive tests, and smoking was defined as the regular use of cigarettes. The study protocol complied with the Declaration of Helsinki and approved by the local ethics committee (Gaziosmanpaşa University Medical School Clinical Trials Ethics Committee, on 25.06.2020 with project number 20-KAEK-150).

\section{Angiographic Findings and Coronary Artery Ostial Diameter Ratio}

All patients underwent elective diagnostic coronary angiography in the presence of positive or suspected result of non-invasive tests. Coronary angiography was performed according to standard clinical protocols via the femoral or radial arteries. Degree of coronary stenosis and ostial diameters of coronary arteries were calculated from digital angiograms for all patients. Ostial diameter of LMCA was calculated in left anterior oblique (LAO) caudal or antero-posterior caudal views, and ostial diameter of RCA was calculated in LAO views. Ostial diameters were calculated manually from digital angiograms within 3 $\mathrm{mm}$ of each coronary ostium, respectively. CAOD-R was calculated by dividing the LMCA ostial diameter by the RCA ostial diameter. Figure 1 demonstrates the schematic illustration of the calculation of CAOD-R. Stenotic CAD was defined as $\geq 50 \%$ stenosis in any segments of the epicardial coronary arteries.
Patients were divided into two groups according to calculated CAOD-R $\leq 1$ or $>1$. The groups were compared regarding the frequency of patients with stenotic $\mathrm{CAD}$, and the association of CAOD-R with the presence of stenotic CAD was investigated.

\section{Statistical Analysis}

Continuous variables were expressed as mean \pm standard deviation whereas categorical variables were expressed as percentages. Normality distribution of continuous variables was tested with the KolmogorovSmirnov test. Student t-test was used to compare the normally distributed continuous variables, and categorical variables were compared with the chi-square test or Fisher's Exact test. To determine the independent predictors of stenotic CAD, a multivariate logistic regression analysis was performed. All variables with $\mathrm{p}<0.05$ in univariate analysis were included in the model. A p-value of $<0.05$ was considered to indicate statistical significance.

\section{Results}

According to calculated CAOD-R, 142 (32.6\%) patients were assigned in the CAOD- $\mathrm{R} \leq 1$ group and 294 (67.4\%) patients were assigned in the CAOD-R $>1$ group. The frequency of patients with DM was significantly higher in the CAOD- $\mathrm{R} \leq 1$ patient group compared to those with CAOD-R $>1$. Importantly, based on the angiographic results, patients with CAOD-R $\leq 1$ had significantly higher frequency of stenotic CAD compared to patients with CAOD-R $>1(61.9 \%$ vs $41.1 \%, p<0.001)$. The groups were
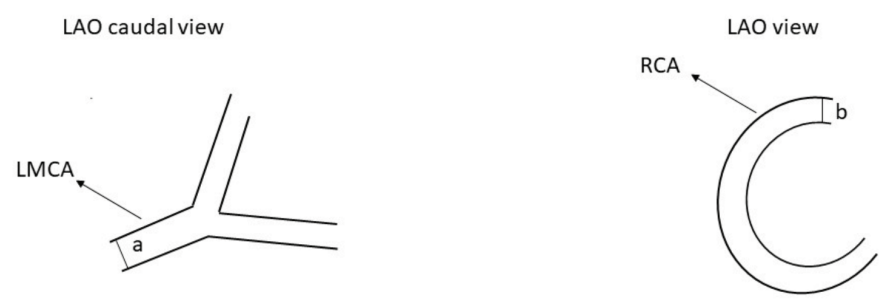

$C A O D-R=a / b$

Figure 1. Schematic illustration of the calculation of coronary artery ostial diameter ratio

LAO: Left anterior oblique, LMCA: Left main coronary artery, CAOD-R: Coronary artery ostial diameter ratio 
similar regarding the coronary dominance pattern. Table 1 demonstrates the clinical, laboratory and angiographic characteristics of patients according to CAOD-R.

When comparing the patients with and without stenotic CAD, patients with stenotic CAD were older, and the frequency of male gender, DM and dyslipidemia were significantly higher in patients with stenotic CAD compared to those without stenotic CAD. Clinical characteristics of patients with and without stenotic CAD are presented in Table 2. In the subgroup analysis of patients with stenotic CAD, while the frequency of left anterior descending (LAD) artery stenosis was significantly higher in the CAOD- $\mathrm{R} \leq 1$ patient group ( $46.6 \%$ vs $38.8 \%$, $\mathrm{p}=0.006)$, the frequency of RCA stenosis was significantly higher in the CAOD-R $>1$ patient group ( $28.9 \%$ vs $18.2 \%$, $\mathrm{p}=0.002$ ). There were no differences between the groups regarding the frequency of stenotic lesions in LMCA and

Table 1. Clinical, laboratory and angiographic characteristics of patients according to coronary artery ostial diameter ratio

\begin{tabular}{|c|c|c|c|}
\hline & $\begin{array}{l}\text { CAOD-R } \leq 1 \\
(n=142)\end{array}$ & $\begin{array}{l}\text { CAOD-R >1 } \\
(n=294)\end{array}$ & $\mathbf{p}$ \\
\hline Age (years) & $51.4 \pm 12.7$ & $52.2 \pm 13.5$ & 0.274 \\
\hline Sex, male, n (\%) & $91(64.1 \%)$ & $193(65.6 \%)$ & 0.114 \\
\hline Hypertension, n (\%) & $53(37.3 \%)$ & $108(36.7 \%)$ & 0.374 \\
\hline Diabetes mellitus, $\mathrm{n}(\%)$ & $41(28.9 \%)$ & $64(21.8 \%)$ & 0.025 \\
\hline Smoking n (\%) & $37(26.1 \%)$ & $85(28.9 \%)$ & 0.475 \\
\hline Previous CAD, $\mathrm{n}(\%)$ & $30(21.1 \%)$ & $67(22.7 \%)$ & 0.817 \\
\hline LVEF (\%) & $58.7 \pm 8.4$ & $56.8 \pm 9.1$ & 0.248 \\
\hline Radial angiography $n,(\%)$ & $26(18.3 \%)$ & $51(17.3 \%)$ & 0.879 \\
\hline WBC count $\left(\times 10^{3} / \mu \mathrm{L}\right)$ & $8.1 \pm 1.4$ & $7.9 \pm 1.3$ & 0.170 \\
\hline Platelet count $\left(\times 10^{3} / \mu \mathrm{L}\right)$ & $255 \pm 55$ & $261 \pm 59$ & 0.408 \\
\hline Hemoglobin (g/dL) & $12.2 \pm 1.7$ & $13.1 \pm 1.8$ & 0.044 \\
\hline Creatinine (mg/dL) & $0.9 \pm 0.15$ & $0.9 \pm 0.13$ & 0.974 \\
\hline ALT (U/L) & $23.5 \pm 9.4$ & $22.2 \pm 8.2$ & 0.571 \\
\hline Total cholesterol (mg/dL) & $183 \pm 40.5$ & $180 \pm 42.7$ & 0.344 \\
\hline LDL-cholesterol (mg/dL) & $107.2 \pm 35.4$ & $97.9 \pm 32.4$ & 0.005 \\
\hline HDL-cholesterol (mg/dL) & $40.3 \pm 8.8$ & $41.2 \pm 7.9$ & 0.082 \\
\hline Right dominance $\mathrm{n},(\%)$ & $111(78.2 \%)$ & $214(72.8 \%)$ & 0.378 \\
\hline Stenotic CAD, n, (\%) & $88(61.9 \%)$ & $121(41.1 \%)$ & $<0.001$ \\
\hline
\end{tabular}

left circumflex artery. Table 3 shows the distribution of stenotic lesions in epicardial coronary arteries according to CAOD-R.

Furthermore, multivariate logistic regression analysis demonstrated that CAOD-R was an independent predictor of the presence of stenotic CAD (odds ratio: 0.824, 95\% confidence interval: $0.721-0.947, \mathrm{p}<0.001)$. Table 4 demonstrates the independent predictors of stenotic CAD in multivariate analysis.

Table 2. Comparison of the clinical characteristics of patients according to the presence or absence of stenotic CAD

\begin{tabular}{|c|c|c|c|}
\hline & $\begin{array}{l}\text { Stenotic } \\
\text { CAD (+) } \\
(n=209)\end{array}$ & $\begin{array}{l}\text { Stenotic } \\
\text { CAD (-) } \\
(n=227)\end{array}$ & $\mathbf{p}$ \\
\hline Age (years) & $55.6 \pm 14.1$ & $50.5 \pm 13.8$ & 0.022 \\
\hline Sex, male, $\mathrm{n}(\%)$ & $158(75.6 \%)$ & $126(55.5 \%)$ & $<0.001$ \\
\hline Hypertension, n (\%) & $85(40.7 \%)$ & $76(33.5 \%)$ & 0.078 \\
\hline Diabetes mellitus, n (\%) & $81(38.7 \%)$ & $24(10.6 \%)$ & $<0.001$ \\
\hline Smoking n (\%) & $67(32.1 \%)$ & $55(24.2 \%)$ & 0.006 \\
\hline Previous CAD, n (\%) & $51(24.4 \%)$ & $46(20.2 \%)$ & 0.441 \\
\hline LVEF (\%) & $57.2 \pm 9.4$ & $57.8 \pm 8.9$ & 0.916 \\
\hline WBC count $\left(\times 10^{3} / \mu L\right)$ & $7.9 \pm 1.4$ & $8.0 \pm 1.5$ & 0.295 \\
\hline Platelet count $\left(\times 10^{3} / \mu \mathrm{L}\right)$ & $261 \pm 58$ & $257 \pm 61$ & 0.108 \\
\hline Hemoglobin (g/dL) & $12.6 \pm 1.8$ & $12.8 \pm 1.8$ & 0.571 \\
\hline Creatinine (mg/dL) & $0.9 \pm 0.1$ & $0.9 \pm 0.1$ & 0.881 \\
\hline $\operatorname{ALT}(\mathrm{U} / \mathrm{L})$ & $22.3 \pm 10.2$ & $22.9 \pm 9.7$ & 0.772 \\
\hline Total cholesterol (mg/dL) & $188 \pm 49.5$ & $175 \pm 47.6$ & 0.044 \\
\hline LDL-cholesterol (mg/dL) & $108.1 \pm 32.2$ & $94.2 \pm 29.6$ & $<0.001$ \\
\hline HDL-cholesterol (mg/dL) & $40.6 \pm 8.4$ & $41.2 \pm 7.2$ & 0.227 \\
\hline
\end{tabular}

Table 3. Distribution of stenotic lesions in epicardial coronary arteries according to coronary artery ostial diameter ratio

\begin{tabular}{|l|l|l|l|} 
Vessel & $\begin{array}{l}\text { CAOD-R } \leq 1 \\
\text { (n=88 stenotic } \\
\text { lesions) }\end{array}$ & $\begin{array}{l}\text { CAOD-R >1 } \\
\text { (n=121 stenotic } \\
\text { lesions) }\end{array}$ & p \\
\hline LMCA, n (\%) & $4(4.5 \%)$ & $5(4.1 \%)$ & 0.984 \\
\hline LAD, n (\%) & $41(46.6 \%)$ & $47(38.8 \%)$ & 0.006 \\
\hline LCX, n (\%) & $27(30.7 \%)$ & $34(28.1 \%)$ & 0.241 \\
\hline RCA, n (\%) & $16(18.2 \%)$ & $35(28.9 \%)$ & 0.002
\end{tabular}

CAOD-R: Coronary artery ostial diameter ratio, LMCA: Left main coronary artery, LAD: Left anterior descending artery, LCX: Left circumflex artery, RCA: Right coronary artery, $n$ : Number 
Table 4. Independent predictors of stenotic CAD in multivariate analysis

\begin{tabular}{|l|l|l|l|}
\hline Variable & $\begin{array}{l}\text { Odds } \\
\text { ratio }\end{array}$ & $\begin{array}{l}\text { \%95 Confidence } \\
\text { Interval }\end{array}$ & $\mathbf{p}$ \\
\hline CAOD-R & 0.824 & $0.721-0.947$ & $<0.001$ \\
\hline LDL-cholesterol & 1.221 & $1.151-1.914$ & $<0.001$ \\
\hline Male, gender & 1.542 & $1.225-2.106$ & $<0.001$ \\
\hline Diabetes mellitus & 2.134 & $1.474-3.261$ & $<0.001$ \\
\hline
\end{tabular}

CAD: Coronary artery disease, CAOD-R: Coronary artery ostial diameter ratio, LDL: Low-density lipoprotein, $n$ : Number

\section{Discussion}

The main finding of our study was that the frequency of stenotic CAD was significantly higher in patients with CAOD-R $\leq 1$ compared to patients with CAOD-R $>1$. In addition, CAOD-R was found to be an independent predictor of stenotic CAD in CCS patients undergoing elective coronary angiography. Also, CAOD-R $\leq 1$ was found to be associated with more frequent LAD artery stenosis and CAOD-R $>1$ was found to be associated with more frequent RCA stenosis. To our knowledge, this is the first study investigating the association of CAOD-R with stenotic CAD in CCS patients.

CAD is characterized by atherosclerotic plaques in the epicardial coronary arteries and coronary angiography is the gold standard diagnostic method for the diagnosis of stenotic coronary atherosclerotic lesions ${ }^{(8)}$. Age, gender, increased blood pressure, DM, obesity and dyslipidemia are well-known traditional risk factors in all stages of CAD from its beginning to symptomatic obstructive $\mathrm{CAD}$; however, there may be numerous other risk factors for coronary atherosclerosis due the dynamic nature and the complex pathophysiology of $\mathrm{CAD}^{(1,3,9)}$. In this sense, anatomic variations in the coronary vascular tree have also been investigated as a risk factor for coronary atherosclerosis and stenotic CAD, and some studies reported a significant association between coronary anatomy and CAD severity ${ }^{(5,10)}$. Moreover, previous studies reported a significant inverse association between coronary artery size and atherosclerotic cardiovascular disease and reported higher frequency of stenotic CAD in small caliber vessels ${ }^{(6,7,11)}$. These findings point out an important link between coronary artery diameter and coronary atherosclerosis, in particular, for stenotic CAD. Considering the pathophysiology of the coronary atherosclerosis, it is sensible to consider that small caliber vessels require smaller plaque burden for stenotic lesions. Also, it has been demonstrated that variations in the size of the coronary arteries may influence the coronary blood flow distribution in the coronary arterial tree that may affect the myocardial perfusion and atherosclerosis ${ }^{(12)}$. Therefore, it seems reasonable to investigate the role of the anatomy of the coronary vasculature in the pathophysiology of the coronary atherosclerosis and stenotic CAD. Nevertheless, these studies generally focused on only diseased vessel diameter and no study has investigated the importance of the ostial diameter of LMCA and RCA. In the present study, we defined a novel parameter named as CAOD-R and demonstrated a significant association between CAOD-R and the presence of stenotic CAD in CCS patients undergoing elective coronary angiography. We found an inverse relationship between CAOD-R and the presence of stenotic CAD, which indicates the importance of ostial size of the main coronary arteries in the distribution of coronary blood flow and severity of coronary atherosclerosis. Besides the predictive value of CAOD-R for stenotic CAD, we also demonstrated that CAOD-R $\leq 1$ was significantly associated with stenotic lesions in LAD artery, and CAOD-R $>1$ was significantly associated with stenotic lesions in RCA. Therefore, CAOD-R may be useful in the prediction of stenotic lesions in different coronary arteries according to its calculated ratio. The findings of the present article are in consistent with the previously published data and provide a novel finding that indicates the clinical importance of diameter ratio of the main coronary arteries for predicting stenotic CAD in the different segments of the coronary vascular tree.

\section{Study Limitations}

The present study has some limitations. First, the size of the each coronary artery was not calculated; however, 
it should be noted that the association between coronary artery size and stenotic CAD has been previously demonstrated. Second, the present study included the patients undergoing elective coronary angiography, and hence the association of CAOD-R with an acute coronary event was not investigated, which may be a topic for another study. Finally, although we considered both traditional and non-traditional cardiovascular risk factors, there may be still some other factors affecting coronary atherosclerosis due to its complex pathophysiology.

\section{Conclusion}

Ostial size of the main coronary arteries may significantly affect the coronary blood flow distribution and coronary hemodynamics that may play a role in the coronary atherosclerosis. In the present study, we demonstrated that as a novel parameter, "CAOD-R" was an independent predictor of stenotic CAD in CCS patients undergoing elective coronary angiography. Additionally, CAOD-R may also be useful in the predicting LAD or RCA stenosis according to its calculated value. Therefore, as an easily calculated parameter from angiographic images, CAOD-R may be useful in the further risk assessment of patients undergoing elective coronary angiography.

\section{Ethics}

Ethics Committee Approval: The study was approved by Gaziosmanpaşa University Medical School Clinical Trials Ethics Committee on 25.06.2020 with project number 20-KAEK-150.

Informed Consent: Retrospective study.

Peer-review: Externally peer-reviewed.

\section{Authorship Contributions}

Concept: M.E., C.E., Design: M.E., C.E., Data Collection or Processing: M.E., C.E., Analysis or Interpretation: M.E., C.E., Literature Search: M.E., C.E., Writing: M.E., C.E.
Conflict of Interest: The authors have no commercial, financial, and other relationships in any way related to the subject of this article that might create any potential conflict of interest.

Financial Disclosure: The authors declared that this study received no financial support.

\section{References}

1. Libby P, Theroux P. Pathophysiology of coronary artery disease. Circulation 2005;111:3481-8.

2. Mozaffarian D, Benjamin EJ, Go AS, et al. Heart disease and stroke statistics - 2016 update: a report from the American Heart Association. Circulation 2016;133:e38-360.

3. Knuuti J, Wijns W, Saraste A, et al. 2019 ESC Guidelines for the diagnosis and management of chronic coronary syndromes Eur Heart J 2020;41:40777.

4. Hajar R. Risk Factors for Coronary Artery Disease: Historical Perspectives. Heart Views 2017;18:109-14.

5. Yan B, Yang J, Fan Y, et al. Association of coronary dominance with the severity of coronary artery disease: a cross-sectional study in Shaanxi Province, China BMJ Open 2018;8:e21292.

6. Nwasokwa ON, Weiss M, Gladstone C, Bodenheimer MM. Effect of coronary artery size on the prevalence of atherosclerosis Am J Cardiol 1996;78:741-6.

7. Zhou FF, Liu YH, Ge PC, et al. Coronary Artery Diameter is Inversely Associated with the Severity of Coronary Lesions in Patients Undergoing Coronary Angiography Cell Physiol Biochem 2017;43:1247-57.

8. Scanlon PJ, Faxon DP, Audet AM, et al. ACC/AHA guidelines for coronary angiography. A report of the American College of Cardiology/American Heart Association Task Force on practice guidelines (Committee on Coronary Angiography). Developed in collaboration with the Society for Cardiac Angiography and Interventions J Am Coll Cardiol 1999;33:1756824.

9. D’Agostino Sr RB, Vasan RS, Pencina MJ, et al. General cardiovascular risk profile for use in primary care. the Framingham Heart Study Circulation 2008;117:743-53.

10. Peng L, Guo X, Gao Y, et al. Impact of right coronary dominance on triplevessel coronary artery disease Medicine (Baltimore). 2018;97:e11685.

11. Makaryus AM, Dhama B, Raince J, et al. Coronary artery diameter as a risk factor for acute coronary syndromes in Asian-Indians. Am J Cardiol 2005;96:778-80

12. Sakamoto S, Takahashi S, Coskun AU, et al. Relation of distribution of coronary blood flow volume to coronary artery dominance. Am J Cardiol 2013;111:1420-4. 